\title{
Immune Thrombocytopenia Secondary to COVID-19: a Systematic Review
}

\author{
Sukrita Bhattacharjee ${ }^{1}$ (D) $\cdot$ Mainak Banerjee $^{2}$
}

Accepted: 10 September 2020 / Published online: 19 September 2020

(C) Springer Nature Switzerland AG 2020

\begin{abstract}
Immune thrombocytopenia, often known as immune thrombocytopenic purpura (ITP), has emerged as an important complication of COVID-19. A systematic review was done to analyze the clinical profile and outcomes in a total of 45 cases of new-onset ITP in COVID-19 patients described in literature until date. A comprehensive approach is essential for diagnosing COVID-19associated ITP after excluding several concomitant factors that can cause thrombocytopenia in COVID-19. Majority of ITP cases $(71 \%)$ were found to be elderly (> 50 years) and $75 \%$ cases had moderate-to-severe COVID-19. Three patients ( $7 \%$ ) were in the pediatric age group. Reports of ITP in asymptomatic COVID-19 patients (7\%) underscore the need for COVID-19 testing in newly diagnosed patients with ITP irrespective of COVID-19 symptoms amid this pandemic. ITP onset occurred in $20 \%$ cases 3 weeks after onset of COVID-19 symptoms, with many reports after clinical recovery. SARS-CoV-2-mediated immune thrombocytopenia can be attributed to the underlying immune dysregulation, susceptibility mutations in SOCS 1, and other mechanisms, including molecular mimicry, cryptic antigen expression, and epitope spreading. No bleeding manifestations were reported in $31 \%$ cases at diagnosis. Severe life-threatening bleeding was uncommon. One case of mortality was attributed to intracranial hemorrhage. Secondary Evans syndrome was diagnosed in one case. Good initial response to short course of glucocorticoids and intravenous immunoglobulin has been found with the exception of delayed lag response in one case. Thrombopoietin receptor agonist usage as a second-line agent has been noted in few cases for short duration with no adverse events. In the relatively short follow-up period, four relapses of ITP were found.
\end{abstract}

Keywords COVID-19 $\cdot$ ITP $\cdot$ Bleeding $\cdot$ Purpura $\cdot$ Hematological complication $\cdot$ Low platelet

\section{Introduction}

A novel coronavirus disease (COVID-19) pandemic, caused by severe acute respiratory syndrome corona virus (SARSCoV-2), has affected over 21 million people worldwide since its inception. Acute respiratory distress syndrome (ARDS), cardiac complications, and thromboembolic events have contributed to majority of the disease mortality. Among

Sukrita Bhattacharjee and Mainak Banerjee contributed equally to this work.

This article is part of the Topical Collection on Covid-19

Sukrita Bhattacharjee

skrtbhttchry@gmail.com

1 Department of Hematology, Institute of Hematology and Transfusion Medicine, Medical College and Hospital, Kolkata, India

2 Institute of Post Graduate Medical Education and Research, Kolkata, India hematological complications, a systemic hyperinflammatory state resembling secondary hemophagocytic lymphohistiocytosis (HLH) and distinct coagulopathy resembling hypercoagulable stage of disseminated intravascular coagulation (DIC) have been reported [1].

The incidence of thrombocytopenia in patients with COVID-19 has been variable across studies. Mild thrombocytopenia has been observed in up to one-third of these patients, with even higher rate in patients with severe disease (57.7\%) compared with nonsevere disease (31.6\%) [2]. A late-onset mild thrombocytopenia (mean time for nadir count from illness onset 28.3 days) for short duration (mean 4.3 days) has also been reported [3]. However, majority did not have any severe degree of thrombocytopenia $\left(<20 \times 10^{9} / \mathrm{L}\right.$ or a sudden drop $>50 \%$ over $24-48 \mathrm{~h}$ ) as can be seen in patients with immune thrombocytopenia irrespective of inciting event [4].

"Immune thrombocytopenic purpura" (ITP), defined by a platelet count $<100 \times 10^{9} / \mathrm{L}$, classically presents with petechiae or purpuric rashes [5]. More recently, a change in the terminology was proposed in favor of "immune 
thrombocytopenia" to recognize that many patients with ITP do not have bleeding symptoms [5]. In this review, both terms have been used interchangeably. ITP has been described following several viral infections, including hepatitis $\mathrm{B} / \mathrm{C}$ viruses (HBV/HCV), cytomegalovirus (CMV), varicella zoster virus (VZV), human immunodeficiency virus (HIV), and most recently, following zika viruses [6]. Multiple cases of ITP secondary to COVID-19 have hitherto been reported [7-29]. Diagnosis of ITP in patients with moderate-to-severe COVID-19 poses a major diagnostic as well as therapeutic challenge to clinicians owing to presence of multiple concomitant conditions including HLH, DIC, sepsis, antibiotic use, heparin prophylaxis, and thromboembolic events.

The aim of this systematic review was to analyze the clinical characteristics, diagnostic work-up, blood count parameters, therapeutic strategies, and outcomes of confirmed COVID-19 patients presenting with new-onset ITP.

\section{Methods}

\section{Search Strategy and Selection of Studies}

A systematic review of literature was performed across PubMed, Google Scholar, and Embase databases until August 25, 2020, using the following keywords as per the PRISMA guidelines: "COVID-19," "immune thrombocytopenic purpura," "thrombocytopenia," "immune cytopenia," "petechiae," "hemorrhage," "bleeding," with interposition of the Boolean operator "AND." The search was conducted by two independent authors (SB and MB). Articles hence identified were further screened. Duplicate articles, articles in nonEnglish language, reviews and comments/communications, and articles not pertaining to new-onset ITP secondary to COVID-19 were excluded. Finally, a total of 23 articles met the eligibility criteria and were included (Fig. 1). The included articles and the number of patients in each article have been summarized in Table 1.

\section{Data Extraction}

The following data were extracted from the included studies: age of the patient at presentation, gender, COVID-19 illness severity, comorbidities, medication use, nadir platelet count, onset of COVID-19 symptoms to diagnosis of ITP, bleeding manifestations of ITP, time to recovery from start of treatment, and clinical outcomes. Mild COVID-19 disease was defined by presence of fever and cough only. Moderate-to-severe COVID-19 disease was defined by presence of dyspnea with pneumonia as evident by crepitations on lung auscultation or radiological evidence. Outcomes to treatment were categorized into two groups: response to treatment and complete response. Response to treatment was defined by documentation of platelet doubling with platelet count reaching at least $>$ $30 \times 10^{9} / \mathrm{L}$. Complete response was noted when platelet reached to $>100 \times 10^{9} / \mathrm{L}$ or baseline count of that patient.

\section{Statistical Analysis}

Statistical analysis was performed using the Statistical Package for Social Sciences (SPSS) 23.0 software program (SPSS Inc., Chicago, IL, USA). Normally distributed data were expressed as mean \pm standard deviation (SD), while nonparametric data were expressed in median (interquartile range, IQR).

\section{Results}

\section{Demographic Profile and Baseline Characteristics}

A total of 45 COVID-19 patients with ITP were included in the final analysis. Fifty-eight percent of patients were males. ITP was reported in one pregnant patient [21]. Median age of the study population was 62 years. Only 3 patients $(7 \%)$ were in the pediatric age group $[18,19,22] ; 32(71 \%)$ patients were above 50 years of age. Needless to say, majority of the study population had wide range of comorbidities. Moderate-tosevere COVID-19 illness was present in $75 \%$ of all patients; mild symptoms were reported in 8 patients $(18 \%)$. Three patients (7\%), who tested positive for COVID-19, did not give any history of fever or other typical flu-like symptoms and were considered to be asymptomatic $[15,21]$. Among patients with individual data of clinical recovery of COVID-19 symptoms $(N=28)$, ITP presented in 6 patients $(21.5 \%)$ in postrecovery period $[7,11,16,19,22,28,29]$. Among these 5 patients, one was negative for COVID-19 at ITP onset [7]. In the French multicenter series [14], SARS-CoV-2 was negative at ITP onset in 2 patients. Data have been presented in Table 2.
Table 1 Articles selected for systematic review

\begin{tabular}{lll}
\hline Number of patients reported by article & Number of articles & Reference number \\
\hline 1 & 16 & {$[7-10,12,13,16,18,19,21-27,29]$} \\
2 & 1 & {$[20]$} \\
3 & 4 & {$[11,15,17,28]$} \\
14 & 1 & {$[14]$} \\
\hline
\end{tabular}


Fig. 1 Article selection process

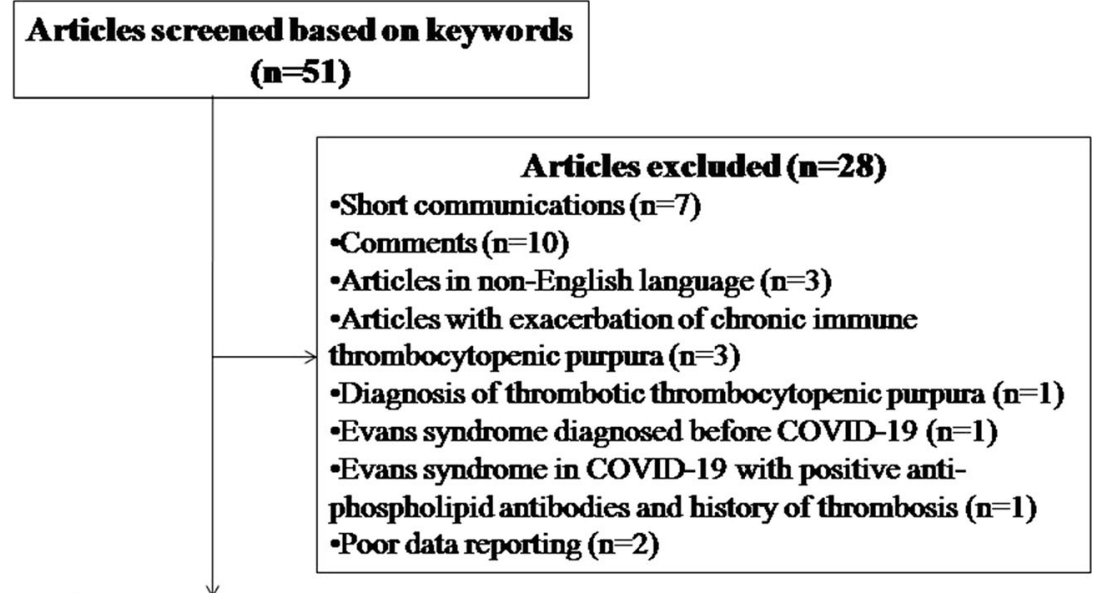

Articles included
\[ (\mathrm{n}=\mathbf{2 3}) \]
-Articles on isolated immune thrombocytopenia $(\mathrm{n}=22)$
-Articles on Evans syndrome $(\mathrm{n}=1)$

\section{ITP Onset and Bleeding Manifestations}

Among all patients, 14 (31\%) patients had no signs of bleeding manifestations at the time of diagnosis. Cutaneous manifestations in form of petechiae/purpura/ ecchymoses were noted in 22 patients; wet purpura was reported in 11 patients. Among other forms of bleeding, epistaxis was commonest. Intracranial hemorrhage was diagnosed in 4 patients $[11,14,23,26]$. Among them, only one mortality has been reported [11]. Two patients had small intraventricular hemorrhage and subarachnoid microhemorrhage in the right frontal lobe, respectively $[23,26]$. Among symptomatic patients with available data $(N=39)$, median day from onset of COVID-19 symptoms to diagnosis of ITP was 13 days, with majority reported in the second and third week. Median nadir platelet count was $5 \times 10^{9} / \mathrm{L}$; only 7 patients $(15.5 \%)$ had nadir count more than $20 \times 10^{9} / \mathrm{L}[14,17,23,24,28]$. Secondary Evans syndrome, as evident by concomitant hemolytic anemia, was diagnosed in one case [29]. Data have been summarized in Table 3.

\section{Treatment and Clinical Outcomes}

Majority $(29 \%)$ of the patients were treated with intravenous immunoglobulin (IVIG) only. Glucocorticoids were used alone in $22 \%$ cases and in combination with IVIG in $24.5 \%$ cases. Thrombopoietin receptor agonists (TP-RA) were used
Table 2 Demographic profile and baseline characteristics of patients with immune thrombocytopenia

\begin{tabular}{ll}
\hline Baseline parameters $(N=45)$ & Value \\
\hline Age (years) (Median $[\mathrm{IQR}])$ & 62 [IQR 44.5-70.5] \\
Age group & $\geq 50$ years $(n=32,71 \%)$ \\
& $18-49$ years $(n=10,22 \%)$ \\
& $<18$ years $(n=3,7 \%)$ \\
Gender & Male $(n=26,58 \%)$ \\
& Female $(n=19,42 \%)$ \\
COVID-19 illness severity & Asymptomatic $(n=3,7 \%)$ \\
& Mild $(n=8,18 \%)$ \\
Clinical recovery from COVID-19 at time of ITP diagnosis $[\mathrm{N}=28]^{\$}$ & Moderate to severe $(n=34,75 \%)$ \\
& Recovery $(n=6,21.5 \%)$ \\
\hline
\end{tabular}

\$ Individual data not available for 28 patients, after excluding 3 patients with no history of COVID-19 symptoms. $\mathrm{IQR}$, inter-quartile range 
Table 3 Clinical characteristics and outcomes in COVID-19 patients with immune thrombocytopenia

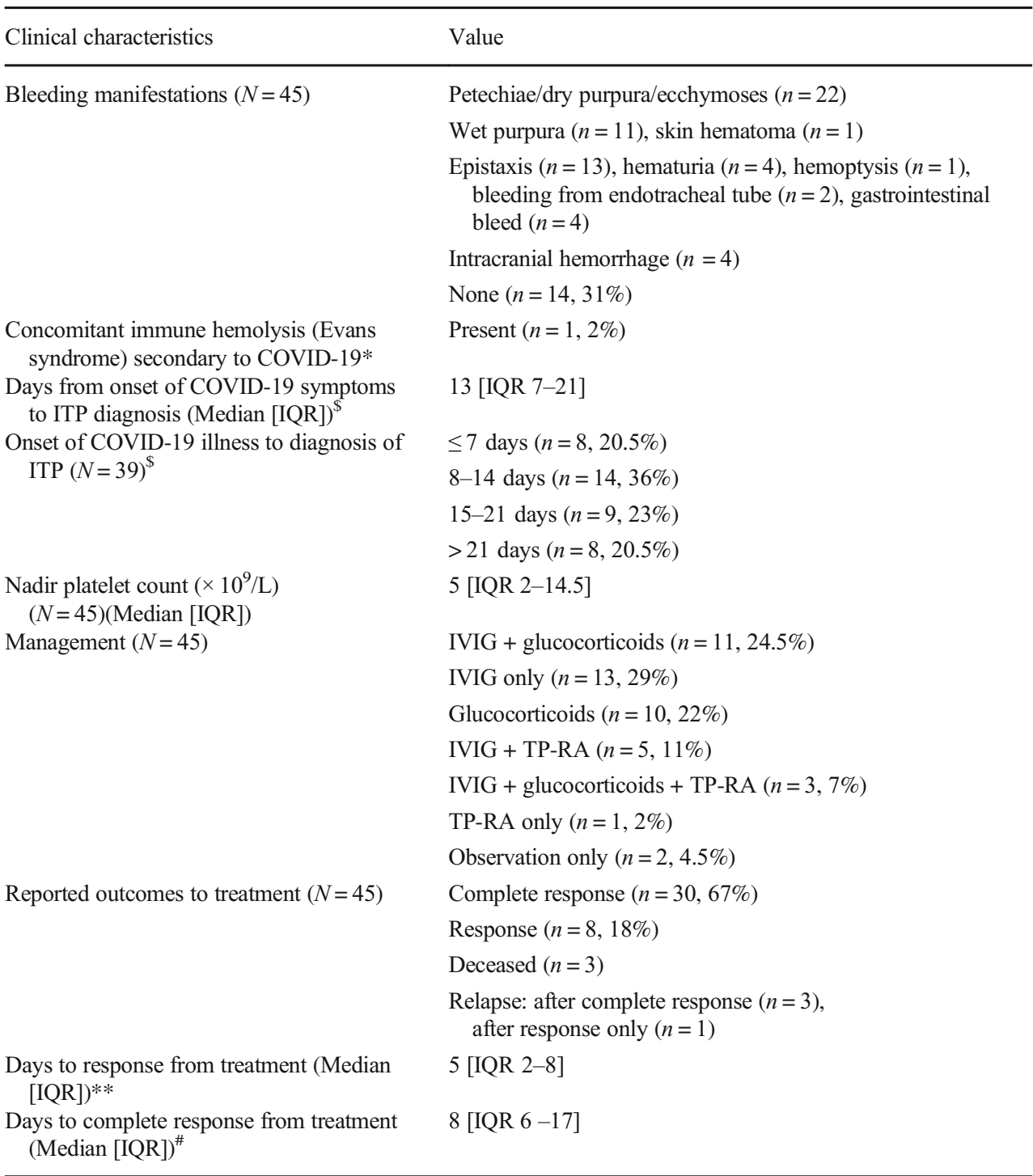

$I Q R$, interquartile range; $I V I G$, intravenous immunoglobulin; TP-RA, thrombopoietin receptor agonist

*Immune hemolysis was diagnosed after clinical recovery from COVID-19 and complete platelet response to IVIG in this patient [29]

\$ Data available for 39 patients after excluding 3 patients with no reported COVID-19 symptoms

**Individual data available for 15 patients

\# Individual data available for 19 patients in 9 patients, either with IVIG $[14,17]$ or both IVIG and glucocorticoids $[14,23,26]$. In one patient, TP-RA was used alone when IVIG was discontinued owing to the development of acute heart failure [17]. In the study population, 26 patients had complete response and 8 patients showed only response to treatment according to study definition. A total of 3 individuals expired: one was due to intracranial hemorrhage [11] and others succumbed to severity of COVID-19 illness after showing platelet response to treatment $[15,27]$. A total of 4 patients (9\%) had relapse following treatment response in the followup period $[13,14]$. Among patients with available individual data, median days to response and complete response were 5 and 8 days, respectively. Data have been presented in Table 3 .

\section{Discussion}

To the best of our knowledge, this is the first comprehensive systematic review of new-onset ITP secondary to COVID-19, comprising 45 cases described in literature until date. We excluded 3 patients with COVID-19 from analysis, who had presented with exacerbation of previously diagnosed chronic or persistent 
ITP [30-32]. Likewise, we excluded one patient who contracted SARS-CoV-2 in post-partum period 3 weeks after being diagnosed with Evans syndrome and treated with rituximab infusion due to poor response to steroids [33]. Another patient with Evans syndrome was excluded due to presence of high titer of antiphospholipid antibodies and past history of venous thrombosis in childhood [34].

\section{Diagnostic Approach}

Immune thrombocytopenia is often a retrospective diagnosis based on exclusion of other possible causes of thrombocytopenia and assessment of the response to treatment [35]. Diagnosing ITP secondary to COVID-19 can be a major challenge for clinicians due to various concomitant conditions or factors associated with COVID-19.

COVID-19 has been associated with a systemic hyperinflammatory state resembling secondary hemophagocytic lymphohistiocytosis [1]. A distinct coagulopathy with high risk of thrombosis has also been observed, which has mandated clinicians to screen moderate-to-severe COVID-19 patients with sepsis-induced coagulopathy (SIC) score or disseminated intravascular coagulation (DIC) score [1]. Both these conditions can cause thrombocytopenia. Evidence of microangiopathic hemolytic anemia with thrombocytopenia must also prompt physicians to exclude thrombotic thrombocytopenic purpura (TTP). Patients should be tested for ADAMTS-13 (a disintegrin and metalloproteinase with a thrombospondin type 1 motif, member 13) activity and ADAMTS-13 inhibitor level, an autoantibody to ADAMTS13. To date, one patient of autoimmune TTP associated with COVID-19 has been reported, who had low ADAMTS-13 activity and very high ADAMTS-13 inhibitor level [36].

In presence of evidence of hemolytic anemia, direct antiglobulin test can clinch the diagnosis of Evans syndrome (ES). ES has been reported following viral infections including hepatitis $\mathrm{C}$, cytomegalovirus, and varicella zoster viruses [37]. In our review, one patient out of total 45 patients was diagnosed with ES secondary to underlying immune dysregulation of COVID19 [29]. This 38-year-old male patient had presented with evidence of immune hemolysis 4 days after being discharged with demonstration of a complete platelet response to treatment of ITP. Of note, authors did not completely rule out the rare possibility of immune hemolytic anemia following IVIG treatment in this case [29].

Due to high risk of thrombosis, heparin prophylaxis has been recommended for all adolescents and adults hospitalized with COVID-19 [1]. Heparin-induced thrombocytopenia (HIT), a well-known clinical entity occurring 5-14 days after heparin exposure, is an important differential for patients presenting with thrombocytopenia after hospitalization. Patients with HIT usually have moderate platelet count $\left(50-70 \times 10^{9} / \mathrm{L}\right)$. They do not develop severe thrombocytopenia $\left(<20 \times 10^{9} / \mathrm{L}\right)$ unless fulminant thrombosis or consumptive coagulopathy develops as a complication [38]. Anti-platelet factor 4 (PF-4)/heparin antibodies or functional assays (serotonin release assay) can be considered if available. However, it is pragmatic to use a clinical scoring system, namely 4Ts score, which incorporates factors, including timing of heparin therapy, complications of thrombosis or thrombocytopenia, and exclusion of other causes [39]. A low 4Ts score $(\leq 3)$ can reliably exclude HIT owing to its high negative predictive value [39]. In the case of clinical suspicion, discontinuation of heparin and consideration to start alternate anti-coagulation strategy (Argatroban, danaparoid) is a safe approach $[8,23]$. Other drugs most commonly implicated to cause thrombocytopenia are quinine, penicillin, tirofiban, and abciximab [40]. In contrast to HIT, drug-induced immune thrombocytopenia (DITP) presents with severe thrombocytopenia $\left(<20 \times 10^{9} / \mathrm{L}\right)$ and bleeding manifestations [40]. Among the patients in review with available data for prior medication use, few received cephalosporins after hospitalization [12, 20, 23]; however, cephalosporin-induced thrombocytopenia is relatively rare. In one patient who received interferon- $\alpha$ and umifenovir, the possibility of DITP could not be ruled out [7]. Management of suspected DITP would be to discontinue the offending agent, send blood for flow cytometry-based testing for drugdependent platelet-reactive antibodies (if available), and consider IVIG or/and glucocorticoids along with platelet transfusions [40].

Viral serologies for hepatitis B (HBV), hepatitis C (HCV), and human immunodeficiency virus (HIV) must be included in the diagnostic work-up. Other infectious agents known to cause ITP are Epstein-Barr virus (EBV), varicella zoster virus (VZV), Parvo-virus B19, cytomegalovirus (CMV), and Helicobacter pylori. If available, testing for these agents can also be considered, as done in few cases $[11,13]$.

Autoimmune profile, including anti-nuclear antibodies (ANA), anti-neutrophil cytoplasmic antibodies (ANCA), rheumatoid factor, anti-cardiolipin, and anti-beta-2 glycoprotein antibodies, should be done. The high incidence of thrombotic events in COVID-19 and reports of high anti-phospholipid antibodies (APLA) in critically ill patients made several researchers to investigate the role of APLA in this coagulopathy [41]. Antiphospholipid syndrome (APLS) can often arise following infectious triggers [42]. However, in view of transient elevation of APLA in various infections or inflammatory states, it is unclear if they represent an epiphenomenon or play a role in coagulopathy in COVID-19. In view of no convincing data at present and unreliability of APLA assay in presence of high C-reactive protein [43], routine APLA testing must be avoided. Mild elevation in ANA titer with nonspecific pattern should also be interpreted cautiously [44]. Of note, positive APLA, low C4, negative antids DNA, and diffusely homogenous high ANA titer (1:320) were found in one COVID-19 patient with Evans syndrome. Past history of venous thrombosis in this patient negates the possibility of secondary APLS due to COVID-19 [34]. Due to a possible association of ITP with multiple myeloma (MM) [45], serum protein electrophoresis has been done in few studies $[8,9]$. It is 
justified to rule out MM, especially for elderly patients with high index of clinical suspicion.

Direct monoclonal antibody immobilization of platelet antigen assay (MAIPA) evaluates presence of platelet autoantibodies on glycoproteins (GP) Ib/IX, Ilb/IIIa, and V bound on the patient platelets [46]. MAIPA, as used in several cases of this review $[8,11,21]$, can be helpful to support the clinical diagnosis of ITP. Indirect MAIPA positivity at ITP onset has also been associated with higher rate of bleeding episodes and development of chronic ITP [47]. However, anti-platelet autoantibodies on platelet surface can be detected in only 40 $60 \%$ of patients [48]. Hence, the diagnosis of ITP should rely on excluding other causes of thrombocytopenia.

Bone marrow aspiration (BMA) study is not essential in the diagnostic work-up for ITP. Increased megakaryocytes can be classically seen with no abnormalities in other cell lineages. BMA should be done if abnormal cells are present in peripheral blood smear. B lymphocyte immunophenotyping is additionally useful in selected cases since lymphoproliferative disorders can often present with ITP and management often involves treatment of the underlying disorder [49]. However, in few cases of chronic lymphocytic leukemia (CLL), management is similar to that of idiopathic ITP [49]. Of note, one patient had a concomitant diagnosis of CLL in the French series [14]. BMA may also help to document hemophagocytosis in COVID-19-associated hyperinflammatory state [1].

ITP was reported in one pregnant patient of this review [21]. Gestational thrombocytopenia, TTP, and HELLP syndrome (hemolysis, elevated liver enzymes, low platelet count) are common differentials to note in this context. A systematic approach to diagnosis of ITP secondary to COVID-19 has been given in Fig. 2.

\section{Profile of Patients with COVID-19-Associated ITP}

COVID-19-associated ITP has been reported more in elderly patients with median age above 60 years of age. Majority of patients were above 50 years of age $(71 \%)$ and had moderateto-severe COVID-19 disease (75\%). Nonetheless, reports of ITP in asymptomatic COVID-19 patients underscore the need for COVID-19 testing in newly diagnosed patients with ITP amid this pandemic. Clinicians should also take note of several reports of ITP in COVID-19 patients in post-recovery period $(21 \%)$.

Clinical presentation of ITP is variable. In this review, 38 $(84.5 \%)$ patients had severe thrombocytopenia (platelet count $<20 \times 10^{9} / \mathrm{L}$ ). ITP patients with severe thrombocytopenia are more likely to have severe bleeding. However, a total of 14 patients $(31 \%)$, including 10 patients with severe thrombocytopenia (out of 38), had no signs of active bleeding manifestations. As opposed to thrombocytopenia due to other causes, for example, bone marrow suppression, the correlation between platelet count and bleeding in ITP is not consistent [50]. Negative antibody results on MAIPA and increased concentration of circulating platelet microparticles (PMP) may partly explain this lack of bleeding in many patients of ITP [47, 51]. Nonetheless, reports of severe bleeding also depend on study population, underlying disease, definition of severe bleeding, and methods of reporting. Of note, no standardized tool for bleeding severity was used for the cases in this review. Nonetheless, intracranial hemorrhage (ICH) was found in 4 patients, with one patient reportedly died of it. In two patients, the microhemorrhage in ventricles or subarachnoid space was not life-threatening. Non-ICH severe forms of bleeding, such as gastrointestinal bleed, metrorrhagia, and hematuria, were reported in 7 patients $[11,14,15,18,25,29]$. Severe forms of bleeding at ITP onset were found to be rare $(<1 \%)$ in a large population-based study comprising 3771 incident ITP patients [52]. However, this data was not exclusive for secondary forms of ITP. Rapidity of platelet fall, as seen in druginduced or allo-immune thrombocytopenia, is another major determinant of bleeding risk [48]. In addition to the dramatic platelet fall, concomitant use of anticoagulation in hospitalized COVID-19 patients can explain the relatively high number of severe bleeding episodes reported in total 11 patients of ITP $(24 \%)$. In case of platelet count $<30 \times 10^{9} / \mathrm{L}$, discontinuation of heparin and intermittent pneumocompression has been recommended in the interim guidance [53].

Onset of ITP has been found more commonly in the second and third week after onset of COVID-19 illness. However, many cases of ITP onset within the first week may be due to the failure of patients to recognize or report onset of first COVID-19 symptom. A considerable proportion of cases were also found to ensue after 3 weeks $(20.5 \%)$. This points towards the myriad of multiple pathophysiological mechanisms by which viruses are known to cause ITP [54].

\section{Pathogenesis of ITP Secondary to COVID-19}

Several possible mechanisms of SARS-CoV-2-mediated thrombocytopenia have already been described $[55,56]$. Mechanisms involve inhibition of platelet synthesis due to direct infection of the bone marrow cells or platelets by the virus (possibly via CD-13 receptors) and dysfunctional marrow microenvironment; virus-mediated liver damage leading to decreased thrombopoietin production; pulmonary endothelial damage followed by platelet aggregation in the lungs, subsequent formation of microthrombi, and platelet consumption; and finally, the destruction of platelets by the immune system manifested as dramatic fall from baseline as described in this review. Potential immune mechanisms of thrombocytopenia have not been adequately discussed until date.

Viral induction of autoimmunity can be explained by various phenomena, including molecular mimicry, cryptic antigen expression, and epitope spreading. The collateral 


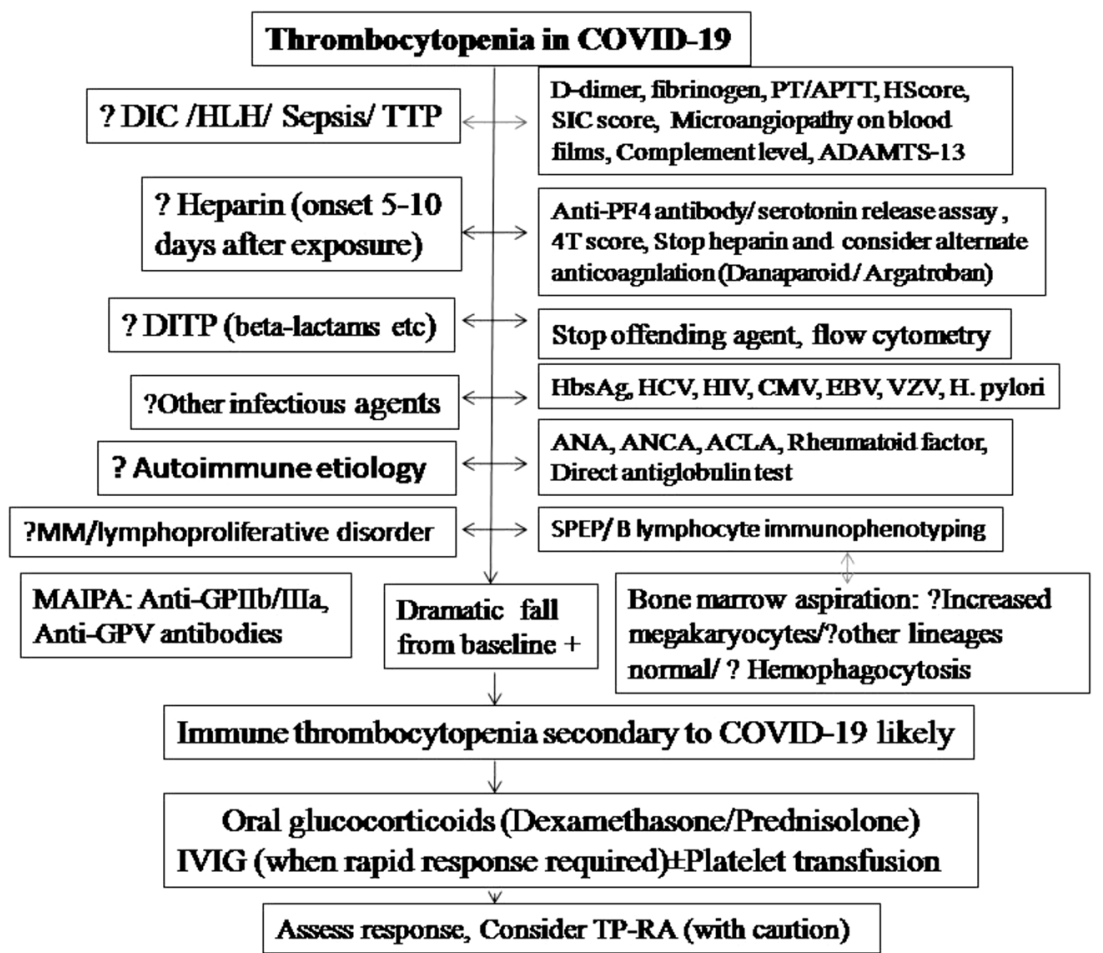

Fig. 2 Diagnostic approach to COVID-19-associated immune thrombocytopenia. DIC, disseminated intravascular coagulation; $H L H$, hemophagocytic lymphohistiocytosis; TTP, thrombotic thrombocytopenic purpura; $P T$, prothrombin time; $A P T T$, activated partial thromboplastin time; ADAMTS-13, a disintegrin and metalloproteinase with a thrombospondin type 1 motif, member 13; PF4, platelet factor 4; DITP, drug-induced thrombocytopenia; $\mathrm{Hbs} A g$, hepatitis B surface antigen;

stimulation of the immune system through certain microbial antigens via "molecular mimicry" has been well described with varicella zoster viruses, HIV, HCV, and H. pylori [57]. This can lead to generation of cross-reactive antibodies to certain glycoproteins on platelet surface. For example, HIV1GP 160/120 antigen can cross-react with the anti-platelet membrane GPIIIa49-66 IgG antibodies [55]. Although antiGP IIb/IIIa, GP-Ib/IX, or GP-V antibodies have been identified in several cases of this review [8, 11, 21], sequence homology between SARS-CoV-2 and platelet components is yet to be identified. Platelets are coated by these anti-platelet antibodies and immune complexes, which result in clearance of platelets by reticuloendothelial system. These antibodies can also inhibit the development of bone marrow megakaryocytes and promote their apoptosis, thus inhibiting platelet production [57]. Bone marrow study in these few selected cases of ITP would reveal low number of megakaryocytes, as seen in one patient of this review, who presented with severe thrombocytopenia (count $2 \times 10^{9} / \mu \mathrm{L}$ ) [7]. COVID-19 nucleic acid test of the bone marrow aspirate was found to be negative in this case [7]. Viruses are capable of inducing expression of cryptic antigens via direct infection of cells. Platelets do not have ACE 2 receptors. However, recent study suggests that
$H C V$, hepatitis $\mathrm{C}$ virus; $H I V$, human immunodeficiency virus; $C M V$, cytomegalovirus; $E B V$, Epstein-Barr virus; $V Z V$, varicella zoster virus; $H$. pylori, Helicobacter pylori; ANA, anti-nuclear antibody; ANCA, antineutrophil cytoplasmic antibody; $A C L A$, anti-cardiolipin antibody; $M M$, multiple myeloma; SPEP, serum protein electrophoresis; MAIPA, monoclonal antibody-specific immobilization of platelet antigen; $I V I G$, intravenous immunoglobulin; $T P-R A$, thrombopoietin receptor agonists

platelets may take up SARS-CoV-2 mRNA independent of ACE2 [58]. Diversification of immune response induced by an antigen to new $\mathrm{T}$ call and/or antibody specificities targeting new target epitopes of same or different antigens is known as epitope spreading. Several factors, including enhanced expression of cryptic antigens under inflammatory milieu and release of self-antigens following tissue damage and B cells, may play a role in induction of this phenomenon. Not surprisingly, it is uncommon for ITP patients to possess single antiplatelet antibody specificity to a particular glycoprotein $[8,11$, 21, 57].

Cytotoxic CD8+ T cells can directly cause platelet lysis, induce the apoptosis of platelets, and inhibit platelet production by megakaryocyte maturation [57]. Low or dysfunctional regulatory CD4+ T cells can also be seen in patients with ITP, pointing towards their possible role [57]. COVID-19 patients with immune dysregulation can have lower levels of regulatory $\mathrm{T}$ cells, which are more decreased in severe cases [59]. This can explain the greater occurrence of ITP in moderate-tosevere COVID-19 cases of this review. Remarkably, greater than one-third of the immunogenic proteins in SARS-CoV-2 have been found to have homology to proteins that are essential to human adaptive immune system [60]. Autoimmunity 
against these proteins and their interactors may impact several functions of adaptive immune system, including MHC class I and class II antigen presentation, cross-presentation of exogenous antigens, and PD-1 signaling [60]. All these may have a role to play in the development of immune cytopenias.

Interestingly, Lee et al. have recently found heterozygous truncation variant in suppressor of cytokine signaling 1 (SOCS 1) in two unrelated COVID-19 patients with autoimmune cytopenias [61]. Heterozygous SOCS 1 loss of function mutations can lead to enhanced interferon signaling and increased immune cell activation, thereby predisposing to immune thrombocytopenia secondary to COVID-19.

C-reactive protein (CRP) can also bind to platelet phosphorylcholine residues, thereby facilitating IgGmediated phagocytic responses against platelets [57]. This perpetuating role of CRP can be implicated in the finding of higher incidence of ITP in patients with moderate-to-severe COVID-19. Pathogenetic mechanisms for COVID-19associated ITP have been summarized in Table 4 .

\section{Management and Outcomes}

Five patients with platelet count $>30 \times 10^{9} / \mathrm{L}$ had minor mucocutaneous bleeding in this review. Among them, spontaneous recovery was noted in one patient on observation only [17], whereas others received glucocorticoids [24, 28]. Although observation alone is recommended as per recent management guidelines for such cases, treatment with glucocorticoids can be considered for those with comorbidities, age $>60$ years or on anticoagulation [35]. IVIG was used alone in majority of these patients in variable doses (400 $\mathrm{mg} / \mathrm{kg} /$ day for 5 days or $1 \mathrm{~g} / \mathrm{kg}$ for $1-3$ days). This can be attributed to the concerns of glucocorticoid use in severe acute respiratory illness in COVID-19 patients [1]. Nonetheless, IVIG is appropriate for patients at risk for severe bleeding because it can produce a platelet count rise within 12-48 h; whereas glucocorticoids usually result in platelet count improvement by 2-5 days [48]. Dexamethasone has been used in standard high doses $40 \mathrm{mg}$ /day for 4 days in majority; $10 \mathrm{mg} /$ day dosing for 10 days was also used in one patient [7]. Intravenous methylprednisolone $(1 \mathrm{mg} / \mathrm{kg} /$ day) or oral prednisolone ( $1 \mathrm{mg} / \mathrm{kg} /$ day) for short duration has also been used. Of note, fear of precipitating ketoacidosis and increased mortality associated with it can be another barrier for its use in usual therapeutic doses in diabetic patients [62]. The American Society of Hematology guideline recommends dexamethasone (40 mg/day for 4 days) or prednisolone ( $1 \mathrm{mg} / \mathrm{kg} /$ day) with tapering (depending on response and for a maximum duration of 6 weeks) [35]. Dexamethasone may be preferred over prednisolone due to finding of increased response rate at 7 days from trials on adults with ITP [35]. However, duration of GC use should be kept at minimum due to concerns for delayed recovery of active COVID-19 disease $[1,53]$. It is reassuring to note that low-dose dexamethasone for maximum 10 days can significantly decrease 28-day mortality significantly for COVID-19 patients
Table 4 Potential mechanisms of immune thrombocytopenia secondary to COVID-19

\begin{tabular}{|c|c|}
\hline Pathogenesis & Mechanisms of thrombocytopenia \\
\hline $\begin{array}{l}\text { Molecular mimicry } \\
\text { - Host generation of cross-reactive anti-platelet anti- } \\
\text { bodies (anti-GP IIb/IIIa, GP-Ib/IX, or GP-V) }\end{array}$ & $\begin{array}{l}\text { - Immune complex formation on platelet surface } \\
\text { leading to clearance by reticuloendothelial system } \\
\text { - Inhibit the development of bone marrow } \\
\text { megakaryocytes and promote their apoptosis }\end{array}$ \\
\hline $\begin{array}{l}\text { Direct viral infection } \\
\text { Local inflammatory milieu }\end{array}$ & $\begin{array}{l}\text { - Expression of cryptic antigen on platelets leading to } \\
\text { recognition by the immune system }\end{array}$ \\
\hline $\begin{array}{l}\text { Release of self-antigens following tissue damage } \\
\text { Local cytokine effects }\end{array}$ & $\begin{array}{l}\text { - Epitope spreading: no single anti-platelet antibody } \\
\text { specificity to a particular glycoprotein }\end{array}$ \\
\hline $\begin{array}{l}\text { Role of B cells } \\
\text { Role of T cells }\end{array}$ & - Direct effect of cytotoxic CD8+ T cells on platelets \\
\hline Immune dysregulation & - Low or dysfunctional regulatory CD4+ T cells \\
\hline $\begin{array}{l}\text { Homology between SARS-CoV-2 immunogenetic } \\
\text { proteins (one-third) and proteins essential to adap- } \\
\text { tive immune system }\end{array}$ & $\begin{array}{l}\text { - Cross-presentation of exogenous antigens } \\
\text { - PD-1 signaling }\end{array}$ \\
\hline $\begin{array}{l}\text { Increased C-reactive protein }(\mathrm{CRP}) \text { in } \\
\text { COVID-19-associated hyperinflammatory state } \\
\text { (perpetuating role) }\end{array}$ & $\begin{array}{l}\text { CRP can bind to platelet phosphorylcholine residues, } \\
\text { thereby facilitating IgG-mediated phagocytic re- } \\
\text { sponses against platelets }\end{array}$ \\
\hline $\begin{array}{l}\text { Heterozygous SOCS } 1 \text { loss of function mutations } \\
\text { (susceptibility locus) }\end{array}$ & $\begin{array}{l}\text { Enhanced interferon signaling and increased immune } \\
\text { cell activation, thereby predisposing to immune } \\
\text { cytopenias }\end{array}$ \\
\hline
\end{tabular}

$G P$, glycoprotein; $S A R S-C o V-2$, severe acute respiratory syndrome corona virus-2; $P D$-1, programmed cell death protein 1; $\operatorname{Ig} G$, immunoglobulin G; SOCS 1, suppressor of cytokine signaling 1 
requiring oxygen or ventilation support [63]. Lack of response or fall of platelets after initial response to short-course GC (without tapering) has been the indication for IVIG or a second GC course in few cases $[8,10,11]$. Lack of sustained response to IVIG monotherapy has also been noted [11]. Thrombopoietin receptor agonists (TP-RA), which can lead to a steady rise in platelet count 1-2 weeks after administration, can also help to avoid rapid recurrences of severe thrombocytopenia [48]. Due to increased potential for thrombotic complications and hepatotoxicity, interim guidance suggests use of TP-RA only as a second-line agent in COVID-19 patients with no evidence of frank DIC [53]. Eltrombopag (25$50 \mathrm{mg}$ ) or romiplostim has been used in total 9 patients (22\%) in the review for a duration from 5 days to 3 weeks; no adverse events were reported [14, 17, 23, 26]. Vincristine single dose along with TP-RA was used in one case due to lack of response to first-line therapies, including IVIG and GC [23]. Authors suggested that high antibody load related to underlying severe COVID-19 and increased platelet consumption or loss of IVIG due to active bleeding might have led to this relative resistance to IVIG treatment [23]. Platelet transfusions have also been used in few of these patients presenting with active bleed.

In the French multicenter series of 14 patients, 3 patients experienced a relapse after a median follow-up period of 60 days (range 30-63 days) [14]. Another patient, initially treated with 2 doses of IVIG, had relapsed at day 28 after discharge in this review [13].

Although specific management guidelines are lacking for Evans syndrome, glucocorticoids are often considered the first-line therapy, with the use of IVIG in severe cases. Second-line therapies include immunosuppressive agents such as rituximab, mycophenolate mofetil, cyclosporine, and cyclophosphamide [37].

This study has certain limitations. First, bias of reporting cases with only very low nadir platelet count cannot be ruled out. Hence, ITP patients with moderate thrombocytopenia may be underrepresented. Second, severity of bleeding could not be graded by a standardized tool. Third, follow-up period of all cases was not adequate to comment on durability of treatment response. Hence, recurrence/relapse data may not be truly reflective of actual data.

\section{Conclusions}

Immune thrombocytopenic purpura (ITP) can occur secondary to COVID-19 infections. A systematic approach is essential to diagnose new-onset ITP after excluding several concomitant factors or conditions that can cause thrombocytopenia in COVID-19. ITP has been found to be more common in elderly and moderate-to-severe COVID-19 patients. Several reports of ITP in asymptomatic COVID-19 patients underscore the need for COVID-19 testing in newly diagnosed patients with ITP amid this pandemic. Clinicians should also take note of several reports of ITP in COVID-19 patients in post-recovery period. SARS-CoV-2-mediated immune thrombocytopenia can be attributed to the underlying immune dysregulation, SOCS 1 mutations, and several other mechanisms, including molecular mimicry, cryptic antigen expression, and epitope spreading. No bleeding was reported in almost one-third of the patients at diagnosis. Severe lifethreatening bleeding has been reported to be uncommon. Good initial response to short course of glucocorticoids and intravenous immunoglobulin has been found with relative resistance in one case. Thrombopoietin receptor agonist usage as a second-line agent has been noted for short duration in few cases with no adverse thrombotic events. In the relatively short follow-up period, four relapses of ITP have been reported.

\section{Compliance with Ethical Standards}

Conflict of Interest The authors declare that they have no conflicts of interest.

\section{References}

1. Bhattacharjee S, Banerjee M, Pal R. COVID-19-associated hemophagocytic lymphohistiocytosis and coagulopathy: targeting the duumvirate [published online ahead of print, 2020 Jun 24]. Indian Pediatr. 2020:S097475591600204.

2. Guan WJ, Ni ZY, Hu Y, Liang WH, Ou CQ, He JX, et al. Clinical characteristics of coronavirus disease 2019 in China. N Engl J Med. 2020;382(18):1708-20. https://doi.org/10.1056/NEJMoa2002032.

3. Chen W, Li Z, Yang B, Wang P, Zhou Q, Zhang Z, et al. Delayedphase thrombocytopenia in patients with coronavirus disease 2019 (COVID-19). Br J Haematol. 2020;190(2):179-84. https://doi.org/ 10.1111/bjh.16885.

4. Sahu KK, Siddiqui AD, Rezaei N, Cerny J. Challenges for management of immune thrombocytopenia during COVID-19 pandemic [published online ahead of print, 2020 Jul 3]. J Med Virol. 2020. https://doi.org/10.1002/jmv.26251.

5. Rodeghiero F, Stasi R, Gernsheimer T, Michel M, Provan D, Arnold DM, et al. Standardization of terminology, definitions and outcome criteria in immune thrombocytopenic purpura of adults and children: report from an international working group. Blood. 2009;113(11):2386-93. https://doi.org/10.1182/blood-2008-07162503.

6. Boyer Chammard T, Schepers K, Breurec S, Messiaen T, Destrem $\mathrm{AL}$, Mahevas M, et al. Severe thrombocytopenia after Zika virus infection, Guadeloupe, 2016. Emerg Infect Dis. 2017;23(4):696-8. https://doi.org/10.3201/eid2304.161967.

7. Chen W, Yang B, Li Z, Wang P, Chen Y, Zhou H. Sudden severe thrombocytopenia in a patient in the recovery stage of COVID-19. Lancet Haematol. 2020;7(8):e624. https://doi.org/10.1016/S23523026(20)30175-7.

8. Deruelle E, Ben Hadj Salem O, Sep Hieng S, Pichereau C, Outin H, Jamme M. Immune thrombocytopenia in a patient with COVID-19 [published online ahead of print, $2020 \mathrm{Jul}$ 16]. Int J Hematol. 2020: 1-6. https://doi.org/10.1007/s12185-020-02943-5. 
9. Hindilerden F, Yonal-Hindilerden I, Sevtap S, Kart-Yasar K. Immune thrombocytopenia in a very elderly patient with Covid19. Front Med (Lausanne). 2020;7:404. Published 2020 Jul 10. https://doi.org/10.3389/fmed.2020.00404.

10. Artru F, Alberio L, Moradpour D, Stalder G. Acute immune thrombocytopaenic purpura in a patient with COVID-19 and decompensated cirrhosis. BMJ Case Rep. 2020;13(7):e236815. Published 2020 Jul 7. https://doi.org/10.1136/bcr-2020-236815.

11. Bomhof G, Mutsaers PGNJ, Leebeek FWG, Boekhorst PAW, Hofland J, Croles FN, et al. COVID-19-associated immune thrombocytopenia. Br J Haematol. 2020;190(2):e61-4. https://doi.org/10. 1111/bjh.16850

12. Humbert S, Razanamahery J, Payet-Revest C, Bouiller K, Chirouze C. COVID-19 as a cause of immune thrombocytopenia. Med Mal Infect. 2020;50(5):459-60. https://doi.org/10.1016/j.medmal.2020. 05.003 .

13. Bennett J, Brown C, Rouse M, Hoffmann M, Ye Z. Immune thrombocytopenia purpura secondary to COVID-19. Cureus. 2020;12(7): e9083. Published 2020 Jul 9. https://doi.org/10.7759/cureus.9083.

14. Mahévas M, Moulis G, Andres E, et al. Clinical characteristics, management and outcome of COVID-19-associated immune thrombocytopenia: a French multicentre series [published online ahead of print, $2020 \mathrm{Jul} \mathrm{17].} \mathrm{Br} \mathrm{J} \mathrm{Haematol.} \mathrm{2020.} \mathrm{https://doi.org/}$ 10.1111/bjh.17024.

15. Ahmed MZ, Khakwani M, Venkatadasari I, Horgan C, Giles H, Jobanputra $\mathrm{S}$, et al. Thrombocytopenia as an initial manifestation of COVID-19; case series and literature review. Br J Haematol. 2020;189(6):1057-8. https://doi.org/10.1111/bjh.16769.

16. Murt A, Eskazan AE, Yilmaz U, Ozkan T, Ar MC. COVID-19 presenting with immune thrombocytopenia: a case report and review of the literature [published online ahead of print, 2020 Jun 4]. J Med Virol. 2020. https://doi.org/10.1002/jmv.26138.

17. Lorenzo-Villalba N, Zulfiqar AA, Auburtin M, et al. Thrombocytopenia in the course of COVID-19 infection. Eur $J$ Case Rep Intern Med. 2020;7(6):001702. Published 2020 May 7. https://doi.org/10.12890/2020 001702.

18. Patel PA, Chandrakasan S, Mickells GE, Yildirim I, Kao CM, Bennett CM. Severe pediatric COVID-19 presenting with respiratory failure and severe thrombocytopenia. Pediatrics. 2020;146(1): e20201437. https://doi.org/10.1542/peds.2020-1437.

19. Rosenzweig JD, McThenia SS, Kaicker S. SARS-CoV-2 infection in two pediatric patients with immune cytopenias: a single institution experience during the pandemic [published online ahead of print, 2020 Jun 21]. Pediatr Blood Cancer. 2020:e28503. https:// doi.org/10.1002/pbc.28503.

20. Revuz S, Vernier N, Saadi L, Campagne J, Poussing S, Maurier F. Immune thrombocytopenic purpura in patients with COVID-19. Eur J Case Rep Intern Med. 2020;7(7):001751. Published 2020 Jun 9. https://doi.org/10.12890/2020_001751.

21. Tang MW, Nur E, Biemond BJ. Immune thrombocytopenia due to COVID-19 during pregnancy. Am J Hematol. 2020;95(8):E191-2. https://doi.org/10.1002/ajh.25877.

22. Tsao HS, Chason HM, Fearon DM. Immune thrombocytopenia (ITP) in a pediatric patient positive for SARS-CoV-2. Pediatrics. 2020;146(2):e20201419. https://doi.org/10.1542/peds.2020-1419.

23. Lévesque V, Millaire É, Corsilli D, Rioux-Massé B, Carrier FM. Severe immune thrombocytopenic purpura in critical COVID-19 [published online ahead of print, $2020 \mathrm{Jul} 1$ 1]. Int J Hematol. 2020:1-5. https://doi.org/10.1007/s12185-020-02931-9.

24. Yang Y, Zhao J, Wu J, Teng Y, Xia X. A rare case of immune thrombocytopenic purpura, secondary to COVID-19 [published online ahead of print, 2020 May 22]. J Med Virol. 2020. https://doi. org/10.1002/jmv.26051.

25. Martincic Z, Skopec B, Rener K, et al. Severe immune thrombocytopenia in a critically ill COVID-19 patient [published online ahead of print, 2020 Aug 6]. Int J Infect Dis. 2020;S12019712(20)30633-0. https://doi.org/10.1016/j.ijid.2020.08.002

26. Zulfiqar AA, Lorenzo-Villalba N, Hassler P, Andrès E. Immune thrombocytopenic purpura in a patient with Covid-19. N Engl J Med. 2020;382(18):e43. https://doi.org/10.1056/NEJMc2010472.

27. Kewan T, Almhana F, Schwartzman L, Daw H, Haddad A, et al. [published online ahead of print, 2020 Aug 12]Int J Lab Hematol. 2020. https://doi.org/10.1111/ijlh.13303.

28. Pascolini S, Granito A, Muratori L, Lenzi M, Muratori P. Coronavirus disease associated immune thrombocytopenia: causation or correlation? [published online ahead of print, 2020 Aug 18]. J Microbiol Immunol Infect. 2020;S1684-1182(20):30203-6. https://doi.org/10.1016/j.jmii.2020.08.006.

29. Li M, Nguyen CB, Yeung Z, Sanchez K, Rosen D, Bushan S. Evans syndrome in a patient with COVID-19. Br J Haematol. 2020;190(2):e59-61. https://doi.org/10.1111/bjh.16846.

30. Hu Z, Chen W, Liang W, Xu C, Sun W, Yi Y. Severe exacerbation of immune thrombocytopenia and COVID-19: the favorable response to corticosteroid-based therapy-a case report [published online ahead of print, 2020 Jun 4]. Ann Hematol. 2020:1-3. https:// doi.org/10.1007/s00277-020-04070-x.

31. Kondo Y, Kaneko Y, Oshige T, et al. Exacerbation of immune thrombocytopaenia triggered by COVID-19 in patients with systemic lupus erythematosus [published online ahead of print, 2020 Aug 5]. Ann Rheum Dis. 2020:annrheumdis-2020-218157. https:// doi.org/10.1136/annrheumdis-2020-218157.

32. Merli M, Ageno W, Sessa F, Salvini M, Caramazza D, Mora B, et al. Recurrence of immune thrombocytopenia at the time of SARS-CoV-2 infection. Ann Hematol. 2020;99(8):1951-2. https://doi.org/10.1007/s00277-020-04130-2.

33. Vadlamudi G, Hong L, Keerthy M. Evans syndrome associated with pregnancy and COVID-19 infection. Case Rep Obstet Gynecol. 2020:2020, 8862545. Published 2020 Aug 18. https:// doi.org/10.1155/2020/8862545.

34. Zarza J, Von Horoch J, Aguayo N, Báez E. Evans syndrome associated with antiphospholipid antibodies in a patient with SARSCOV-2 infection [published online ahead of print, 2020 Aug 21]. Hematol Transfus Cell Ther. 2020. https://doi.org/10.1016/j.htct. 2020.08.003.

35. Neunert C, Terrell DR, Arnold DM, et al. American Society of Hematology 2019 guidelines for immune thrombocytopenia. Blood Adv. 2019;3(23):3829-3866. Blood Adv. 2020;4(2):252. doi:https://doi.org/10.1182/bloodadvances.2019001380

36. Hindilerden F, Yonal-Hindilerden I, Akar E, Kart-Yasar K. Covid19 associated autoimmune thrombotic thrombocytopenic purpura: report of a case [published online ahead of print, $2020 \mathrm{Jul} 5$ ]. Thromb Res. 2020;195:136-8. https://doi.org/10.1016/j.thromres. 2020.07.005.

37. Jaime-Pérez JC, Aguilar-Calderón PE, Salazar-Cavazos L, GómezAlmaguer D. Evans syndrome: clinical perspectives, biological insights and treatment modalities. J Blood Med. 2018;9:171-84. Published 2018 Oct 10. https://doi.org/10.2147/JBM.S176144.

38. Arepally GM. Heparin-induced thrombocytopenia. Blood. 2017;129(21):2864-72. https://doi.org/10.1182/blood-2016-11709873.

39. Cuker A, Gimotty PA, Crowther MA, Warkentin TE. Predictive value of the 4Ts scoring system for heparin-induced thrombocytopenia: a systematic review and meta-analysis. Blood. 2012;120(20): 4160-7. https://doi.org/10.1182/blood-2012-07-443051.

40. Arnold DM, Nazi I, Warkentin TE, Smith JW, Toltl LJ, George JN, et al. Approach to the diagnosis and management of drug-induced immune thrombocytopenia. Transfus Med Rev. 2013;27(3):13745. https://doi.org/10.1016/j.tmrv.2013.05.005.

41. Parodi A, Gasparini G, Cozzani E. Could antiphospholipid antibodies contribute to coagulopathy in COVID-19? J Am Acad 
Dermatol. 2020;83(3):e249. https://doi.org/10.1016/j.jaad.2020.06. 003.

42. Linnemann B. Antiphospholipid syndrome - an update. Vasa. 2018;47(6):451-64. https://doi.org/10.1024/0301-1526/a000723.

43. Mehta S, Bhandari S, Mehta S. Cautious interpretation of antiphospholipid antibodies in COVID-19. Clin Chim Acta. 2020;509:166. https://doi.org/10.1016/j.cca.2020.06.024.

44. Selmi C, Ceribelli A, Generali E, Scirè CA, Alborghetti F, Colloredo G, et al. Serum antinuclear and extractable nuclear antigen antibody prevalence and associated morbidity and mortality in the general population over 15 years. Autoimmun Rev. 2016;15(2): 162-6. https://doi.org/10.1016/j.autrev.2015.10.007.

45. Gupta V, Hegde UM, Parameswaran R, Newland AC. Multiple myeloma and immune thrombocytopenia. Clin Lab Haematol. 2000;22(4):239-42. https://doi.org/10.1046/j.1365-2257.2000. 00125.x.

46. Porcelijn L, Huiskes E, Oldert G, Schipperus M, Zwaginga JJ, de Haas M. Detection of platelet autoantibodies to identify immune thrombocytopenia: state of the art. Br J Haematol. 2018;182(3): 423-6. https://doi.org/10.1111/bjh.15404.

47. Grimaldi D, Canouï-Poitrine F, Croisille L, Lee K, RoudotThoraval F, Languille L, et al. Antiplatelet antibodies detected by the MAIPA assay in newly diagnosed immune thrombocytopenia are associated with chronic outcome and higher risk of bleeding. Ann Hematol. 2014;93(2):309-15. https://doi.org/10.1007/ s00277-013-1855-5.

48. Arnold DM. Bleeding complications in immune thrombocytopenia. Hematology Am Soc Hematol Educ Program. 2015;2015:237-42. https://doi.org/10.1182/asheducation-2015.1.237.

49. Durani U, Go RS, Kay NE. Immune-mediated hemolytic anemia and thrombocytopenia in clonal B-cell disorders: a review. Clin Adv Hematol Oncol. 2018;16(10):670-6.

50. Neunert C, Noroozi N, Norman G, Buchanan GR, Goy J, Nazi I, et al. Severe bleeding events in adults and children with primary immune thrombocytopenia: a systematic review. J Thromb Haemost. 2015;13(3):457-64. https://doi.org/10.1111/jth.12813.

51. Boulware R, Refaai MA. Why do patients with immune thrombocytopenia (ITP) experience lower bleeding events despite thrombocytopenia? Thromb Res. 2020;187:154-8. https://doi.org/10.1016/ j.thromres.2020.01.020.

52. Moulis G, Palmaro A, Montastruc JL, Godeau B, Lapeyre-Mestre M, Sailler L. Epidemiology of incident immune thrombocytopenia: a nationwide population-based study in France. Blood. 2014;124(22):3308-15. https://doi.org/10.1182/blood-2014-05578336.

53. Pavord S, Thachil J, Hunt BJ, Murphy M, Lowe G, Laffan M, et al. Practical guidance for the management of adults with immune thrombocytopenia during the COVID-19 pandemic. Br J
Haematol. 2020;189(6):1038-43. https://doi.org/10.1111/bjh. 16775.

54. Assinger A. Platelets and infection - an emerging role of platelets in viral infection. Front Immunol. 2014;5:649. Published 2014 Dec 18. https://doi.org/10.3389/fimmu.2014.00649.

55. Xu P, Zhou Q, Xu J. Mechanism of thrombocytopenia in COVID19 patients. Ann Hematol. 2020;99(6):1205-8. https://doi.org/10. 1007/s00277-020-04019-0.

56. Zhang Y, Zeng X, Jiao Y, Li Z, Liu Q, Ye J, et al. Mechanisms involved in the development of thrombocytopenia in patients with COVID-19 [published online ahead of print, 2020 Jun 5]. Thromb Res. 2020;193:110-5. https://doi.org/10.1016/j.thromres.2020.06. 008.

57. Li C, Li J, Ni H. Crosstalk between platelets and microbial pathogens. Front Immunol. 2020;11:1962. Published 2020 Aug 7. https://doi.org/10.3389/fimmu.2020.01962.

58. Manne BK, Denorme F, Middleton EA, et al. Platelet gene expression and function in COVID-19 patients [published online ahead of print, 2020 Jun 23]. Blood. 2020:blood.2020007214. https://doi. org/10.1182/blood.2020007214.

59. Qin C, Zhou L, Hu Z, Zhang S, Yang S, Tao Y, et al. Dysregulation of immune response in patients with coronavirus 2019 (COVID-19) in Wuhan, China. Clin Infect Dis. 2020;71(15):762-8. https://doi. org/10.1093/cid/ciaa248.

60. Lyons-Weiler J. Pathogenic priming likely contributes to serious and critical illness and mortality in COVID-19 via autoimmunity. J Transl Autoimmun. 2020;3:100051. Published 2020 Apr 9. https://doi.org/10.1016/j.jtauto.2020.100051.

61. Lee PY, Platt CD, Weeks S, et al. Immune dysregulation and multisystem inflammatory syndrome in children (MIS-C) in individuals with haploinsufficiency of SOCS1 [published online ahead of print, 2020 Aug 24]. J Allergy Clin Immunol. 2020;S0091-6749(20): 31170-2. https://doi.org/10.1016/j.jaci.2020.07.033.

62. Pal R, Banerjee M, Yadav U, Bhattacharjee S. Clinical profile and outcomes in COVID-19 patients with diabetic ketoacidosis: a systematic review of literature [published online ahead of print, 2020 Aug 18]. Diabetes Metab Syndr. 2020;14(6):1563-9. https://doi. org/10.1016/j.dsx.2020.08.015.

63. RECOVERY Collaborative Group, Horby P, Lim WS, et al. Dexamethasone in hospitalized patients with Covid-19 - preliminary report [published online ahead of print, $2020 \mathrm{Jul} \mathrm{17].} \mathrm{N} \mathrm{Engl} \mathrm{J}$ Med. 2020:NEJMoa2021436. https://doi.org/10.1056/ NEJMoa2021436.

Publisher's Note Springer Nature remains neutral with regard to jurisdictional claims in published maps and institutional affiliations. 